\title{
MULTIPLEXED CLUSTERED-DOT HALFTONE WATERMARKS USING BI-DIRECTIONAL PHASE MODULATION AND DETECTION
}

\author{
Basak Oztan* and Gaurav Sharma \\ ECE Dept., University of Rochester, Rochester, NY 14627-0126 USA \\ basak.oztan@ieee.org, gaurav.sharma@rochester.edu
}

\begin{abstract}
We present a method for embedding and detection of visual watermark patterns in printed images that use clustered-dot halftones in the printing process. The method allows two independent watermark patterns to be multiplexed, i.e. embedded in the same region of the printed image, thereby offering an improvement over prior techniques. The watermark patterns are embedded via phase modulation along the two periodicity directions of the halftone. Each embedded pattern can be visually detected, without interference from the other watermark, when the printed image, or a scan thereof, is overlaid with a decoder mask consisting of periodic lines oriented along the corresponding halftone periodicity direction. We experimentally demonstrate that our proposed multiplexing technique is effective. We also present analysis that demonstrates that the embedding has minimal visual impact and explains the pattern observed in the watermark detection process.
\end{abstract}

Index Terms - Halftone watermarks, clustered-dot halftones, halftone phase modulation, print watermarks

\section{INTRODUCTION}

Hardcopy watermarks have been extensively used for content authentication and counterfeit protection for over seven centuries [1, 2]. Paper watermarks, which represent the most prevalent form of hardcopy watermarks, are embedded within the printing substrate at the time of manufacture [2, Chap. 3]. As digital printing methods become commonplace, the flexibility that these methods offer over conventional analog printing has also been exploited in order to develop a number of alternative digital watermarking methods for hardcopy prints.

Halftoning is typically utilized for the reproduction of images in most digital printing systems [3] and many of the digital hardcopy watermarking techniques exploit the characteristics of the halftoning process for embedding watermarks in the printed images [4-10]. A majority of these methodologies [4-8] mimic the conventional paper watermarks and utilize visual watermark patterns such as bi-level text images or logos. The watermarks are embedded in the form of changes in the halftone structures that are normally imperceptible but can be revealed by appropriate detection methods.

In this paper, we present a technique that enables embedding and detection of two independent visual watermark patterns in the same region of a halftone image. Our technique is specifically designed for periodic clustered-dot halftones that are widely utilized in electrophotographic (laser) and lithographic (offset) printing. Based on the analogy with multiplexing in communications, we refer to

* Basak Oztan is now with the Dept. of Computer Science, Rensselaer Polytechnic Institute, Troy, NY, 12180-3590, USA.

This work is partly supported by a grant from New York State Office of Science, Technology, and Academic Research (NYSTAR) through the Center for Electronic Imaging Systems (CEIS) of University of Rochester. the technique as multiplexed clustered-dot halftone watermarking. The method operates by exploiting the flexibility offered by the 2D periodicity of clustered-dot halftones and embeds the watermarks as directional local phase variations along the two periodicity directions of the halftone. The embedded patterns affect the visual appearance of the halftone image only minimally but can be detected independently by using two decoder masks that consist of periodic lines oriented along the corresponding directions. The rest of the paper is organized as follows: in Section 2 we describe multiplexed halftone watermarking using bi-directional phase modulation, then in Section 3 we present and analyze the watermark detection process, experimental results are shown in Section 4, and finally in Section 5 we present conclusions.

\section{MULTIPLEXED HALFTONE WATERMARKING USING BI-DIRECTIONAL PHASE MODULATION}

Denoting 2-D spatial coordinates by a vector $\mathbf{x}=[x, y]^{T}$, our objective can be stated as follows: Given a continuous tone (contone) image $I(\mathbf{x})$ and two independent visual watermark patterns $w_{1}(\mathbf{x})$ and $w_{2}(\mathbf{x})$, we wish to obtain a halftone image $I^{h}(\mathbf{x})$ such that at normal viewing distance, a print of $I^{h}(\mathbf{x})$ has a visual appearance that closely approximates $I(\mathbf{x})$ and additionally the watermark patterns $w_{1}(\mathbf{x})$ and $w_{2}(\mathbf{x})$ are recoverable from the print.

Specifically, we will consider clustered-dot, or amplitude modulated (AM), halftones. A halftone image that meets the requirement that its visual appearance at normal viewing distance closely approximates $I(\mathbf{x})$, but does not carry the watermark patterns, can be obtained via a screening process [11] that compares the image $I(\mathbf{x})$ against a periodic threshold function $T(\mathbf{x})$ as

$$
I^{h}(\mathbf{x})= \begin{cases}1 & \text { if } I(\mathbf{x})<T(\mathbf{x}), \\ 0 & \text { otherwise }\end{cases}
$$

where we adopt the convention that the values 1 and 0 correspond, respectively, to whether ink/toner is, or is not, deposited at the pixel position $\mathrm{x}$.

As illustrated in Fig. 1, in general the halftone spatial periodicity is determined by a periodic tiling of the plane, which is equivalently represented by two fundamental screen frequency vectors $\mathbf{f}_{1}$ and $\mathbf{f}_{2}$ that are commonly defined in polar coordinates by their magnitudes $\left|\mathbf{f}_{1}\right|$ and $\left|\mathbf{f}_{2}\right|$ and angles $\phi_{1}$ and $\phi_{2}$, respectively. Note that $\left|\mathbf{f}_{1}\right|$ and $\left|\mathbf{f}_{2}\right|$, and $\phi_{1}$ and $\phi_{2}$ are equal to each other for typical orthogonal halftone screens.

A simple and useful analytic threshold function for generating periodic clustered-dot halftones is obtained as a generalization of [12,13] as [14] $T(\mathbf{x})=\cos \left(2 \pi \mathbf{f}_{1}^{T} \mathbf{x}\right)+\cos \left(2 \pi \mathbf{f}_{2}^{T} \mathbf{x}\right)$. In order to embed the watermark patterns, we modulate the phase of the cosine functions as

$$
T(\mathbf{x})=\cos \left(2 \pi \mathbf{f}_{1}^{T} \mathbf{x}+\Psi_{1}(\mathbf{x})\right)+\cos \left(2 \pi \mathbf{f}_{2}^{T} \mathbf{x}+\Psi_{2}(\mathbf{x})\right),
$$




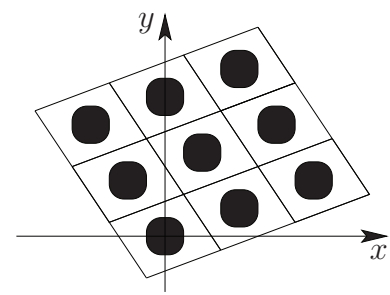

(a)

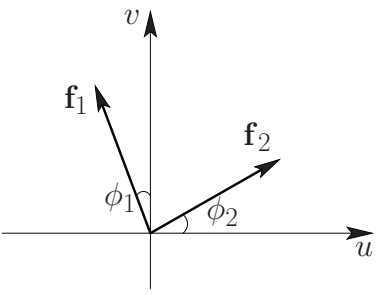

(b)
Fig. 1. Subfigure (a) shows a periodic clustered-dot halftone and (b) shows the corresponding fundamental screen frequencies.

where $\Psi_{1}(\mathbf{x})$ and $\Psi_{2}(\mathbf{x})$ depend on the watermark patterns $w_{1}(\mathbf{x})$ and $w_{2}(\mathbf{x})$ and allow phase shifts along the orientations $\phi_{1}$ and $\phi_{2}$, respectively. Note that the use of an analytic threshold function is similar to the continuous phase-modulated (CPM) watermarks [6]. However, the summation form of the function chosen here is different from the version adopted for CPM because it allows for independent embedding and detection ${ }^{1}$. Specifically, we assume that $\Psi_{i}(\mathbf{x})=\tilde{w}_{i}(\mathbf{x})$, where $\tilde{w}_{i}(\mathbf{x})$ is obtained by normalizing $w_{i}(\mathbf{x})$ between 0 and 1 after a potential blurring to eliminate discontinuities that typically cause visible artifacts [6]. This ensures that in regions where $\tilde{w}_{i}(\mathbf{x})$ equals 1 , the halftone spots are shifted by half a period along the corresponding periodicity direction $\phi_{i}+\pi / 2$.

Characterization of the phase-modulated halftone as a function of the embedded watermark patterns and the cover image halftone is useful in order to understand both the visual impact of the embedding process and the detection process that we describe in the next section. For this purpose, we obtain a Fourier representation for the phase-modulated halftone. Denoting the spatial displacements (in halftone cell units) corresponding to the phase modulation $\Psi_{i}(\mathbf{x})$ by $d_{i}(\mathbf{x})=\Psi_{i}(\mathbf{x}) / 2 \pi$, the phase-modulated halftone can be represented by decomposing the contone image in the form of a sifting integral [15] and combining it with the Fourier series representation for a constant phase-shifted (2-fold) periodic halftone for a constant gray-level image [16] as

$$
\begin{aligned}
I^{h}(\mathbf{x})=\sum_{n_{1}=-\infty}^{\infty} \sum_{n_{2}=-\infty}^{\infty} c_{n_{1}, n_{2}}^{\tilde{I}(\mathbf{x})} & \exp \left\{j 2 \pi \left(n_{1}\left(\mathbf{f}_{1}^{T} \mathbf{x}-d_{1}(\mathbf{x})\right)\right.\right. \\
& \left.\left.+n_{2}\left(\mathbf{f}_{2}^{T} \mathbf{x}-d_{2}(\mathbf{x})\right)\right)\right\}
\end{aligned}
$$

where

$$
c_{n_{1}, n_{2}}^{\mu}=\frac{1}{|\mathcal{U}|} \int_{\mathcal{U}} s(\mathbf{x} ; \mu) \exp \left\{-j 2 \pi\left(n_{1} \mathbf{f}_{1}^{T} \mathbf{x}+n_{2} \mathbf{f}_{2}^{T} \mathbf{x}\right)\right\} d \mathbf{x}
$$

are the Fourier series coefficients for the halftone spot function $s(\mathbf{x} ; \mu)$ for an image gray-level $\mu=I\left(\mathbf{x}-d_{1}(\mathbf{x})-d_{2}(\mathbf{x})\right), \mathcal{U}$ is the 2-D halftone cell representing the periodicity of the halftone and $|\mathcal{U}|$ is its area. The modulation in phase causes variations in the instantaneous frequencies around a narrow region about the corresponding fundamental screen frequencies. Thus, the low frequency content (or the visual appearance) is not changed by the embedded watermarks (Also see [6] for the analysis of visual distortion caused by halftone phase modulation). This can also be seen in Fig. 2, which shows an enlarged view of the log-magnitude Fourier spectrum of the bi-directional phase modulation based watermarked Chapel halftone that is used in our experiments. Note that since the two watermarks are different, they yield different patterns around their corresponding fundamental screen frequencies.

${ }^{1}$ Although two independent watermark patterns can be embedded using the product form described in [6], the embedded watermarks cannot be detected independently in that setting.

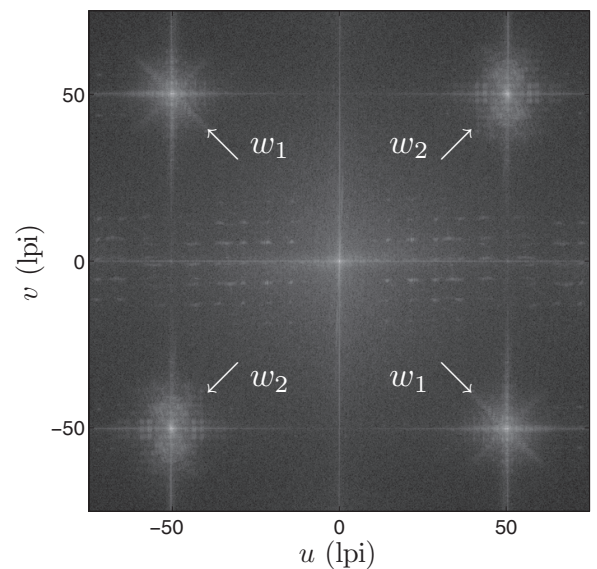

Fig. 2. Enlarged view of the log-magnitude Fourier spectrum of scanned Chapel halftone, where two independent watermarks $w_{1}$ and $w_{2}$ are embedded as local phase variations along the directions indicated by arrow and text labels.

\section{DETECTION OF THE EMBEDDED WATERMARK PATTERNS}

The watermark pattern $w_{i}, i \in\{1,2\}$, is revealed by overlaying the printed image with a transparency bearing a constant gray-level (non-modulated) periodic line screen [11], i.e. an alternating pattern of transparent and opaque (black) lines, that has the fundamental screen frequency $\mathbf{f}_{i}$. If the magnitudes of the screen frequencies $\left|\mathbf{f}_{1}\right|$ and $\left|\mathbf{f}_{2}\right|$ are not equal to each other (for example, as in nonorthogonal halftone screens [11]), two decoder masks are needed. Otherwise, a single transparency mask can be used for the detection of the two watermarks (by adjusting the orientation manually). The $i^{t h}$ decoder mask $I_{m_{i}}^{h}(\mathbf{x})$ can be obtained, via a screening process, by using the threshold function $T_{m_{i}}(\mathbf{x})=\cos \left(2 \pi \mathbf{f}_{i}^{T} \mathbf{x}\right)$. The area coverage of the masks is typically $50 \%$ to allow maximal contrast in the detected watermark [6].

In order to characterize the detection process, we also use a Fourier representation for the decoder masks. The decoder mask $I_{m_{i}}^{h}(\mathbf{x})$ is a (1-fold) periodic function that can be represented in the form of Fourier series as $I_{m_{i}}^{h}(\mathbf{x})=\sum_{n=-\infty}^{\infty} 0.5 \operatorname{sinc}(0.5 n)$ $\times \exp \left\{j 2 \pi n \mathbf{f}_{i}^{T} \mathbf{x}\right\}$, where an area coverage of $50 \%$ is assumed. The overlay of the phase-modulated halftone $I^{h}(\mathbf{x})$ and the decoder mask $I_{m_{i}}^{h}(\mathbf{x}), i \in\{1,2\}$, can be obtained by multiplying (3) with the corresponding Fourier series representation for the mask. For simplicity, we assume the halftone spots are square-shaped for which the Fourier series coefficients in (3) are obtained as

$$
c_{n_{1}, n_{2}}^{\tilde{I}(\mathbf{x})}=\tilde{I}(\mathbf{x}) \operatorname{sinc}\left(n_{1} \sqrt{\tilde{I}(\mathbf{x})}\right) \operatorname{sinc}\left(n_{2} \sqrt{\tilde{I}(\mathbf{x})}\right),
$$

where the contone image values $I(\mathbf{x})$ are assumed to be normalized between 0 and 1 . Since $\mathbf{f}_{1}$ and $\mathbf{f}_{2}$ are typically separated by large angles in practice (for example, by 90-deg in orthogonal halftones), integer linear combinations of $\mathbf{f}_{1}$ and $\mathbf{f}_{2}$ do not appear at low frequencies. Therefore, the low frequency components in the resulting overlay depend, along with the image values of the cover image and the decoder mask, only on $d_{i}(\mathbf{x})$ and, therefore, $w_{i}(\mathbf{x})$ the watermark pattern embedded along the orientation $\phi_{i}$. Note that the modulation corresponding to the other watermark pattern continues to stay at high frequencies and is therefore not visible when decoding with the decoder mask $I_{m_{i}}^{h}(\mathbf{x})$ for the $i^{t h}$ watermark. In other 
words, the decoder mask $I_{m_{i}}^{h}(\mathbf{x})$ acts as a frequency demodulator by selectively translating the frequency components around the corresponding screen frequency $\mathbf{f}_{i}$ (and its harmonics) to low frequencies. The low frequency components that corresponds to the visual appearance can be shown to be

$$
\sum_{n=-\infty}^{\infty} 0.5 \tilde{I}(\mathbf{x}) \operatorname{sinc}(0.5 n) \operatorname{sinc}(n \sqrt{\tilde{I}(\mathbf{x})}) \exp \left\{-j 2 \pi n d_{i}(\mathbf{x})\right\} .
$$

In order to relate this expression to the visual appearance of the detected watermark, we use a 1-D spatial domain model [6], as shown in Fig. 3, to obtain analytical expressions for the overlay reflectance as a function of the cover image and the phase modulation signal. We assume a (locally) constant phase modulation signal $\Psi(x)=\psi$ that shifts the halftone spots in the phase-modulated halftone by a constant displacement of $d=\psi / 2 \pi$ in halftone cell units, where $\mu$ denotes the halftone area coverage. In order to detect the change in phase, the modulated halftone is overlaid with the decoder mask with an area coverage of $50 \%$.

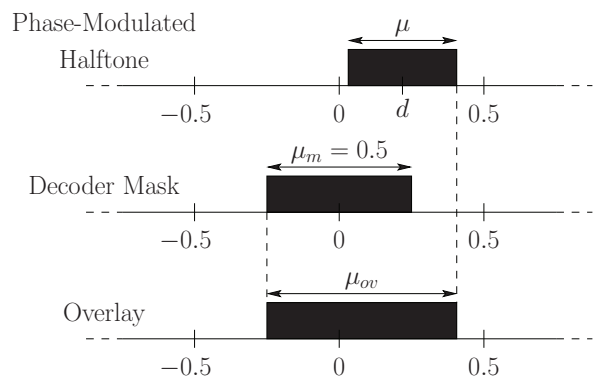

Fig. 3. Model for computing the average reflectance of the overlay.

In this scenario, the reflectance $R_{o v}(\mu, d)$ of the overlay is readily obtained for $\mu \leq 0.5$ (the case for $\mu>0.5$ can be obtained through mirror symmetry) as [6] $R_{o v}(\mu, d)=1-\mu_{o v}(\mu, d)$, where

$$
\mu_{o v}(\mu, d)= \begin{cases}0.5 & \text { if }|d| \leq \frac{0.5-\mu}{2} \\ |d|+\frac{\mu+0.5}{2} & \text { if } \frac{0.5-\mu}{2} \leq|d| \leq \frac{\mu+0.5}{2} \\ \mu+0.5 & \text { if } \frac{\mu+0.5}{2} \leq|d| \leq 0.5\end{cases}
$$

Using this expression, one can determine the spatial variations in reflectance observed in the overlay created in the detection process for a slowly varying modulating pattern $d(x)$ as a function of the halftone image area coverage $\mu(x)$ (or equivalently the image graylevel $I(x))$ as $R_{o v}(\mu(x), d(x))$.

The effect of the alignment between the cover halftone image and the decoder mask can be incorporated in this computation by writing $d(x)=\Psi(x) / 2 \pi+d_{0}$, where $d_{0} \in[-0.5,0.5]$ represents the initial displacement between the cover image halftone and the decoder mask (in halftone cell width units) [6]. Clearly the overlay reflectance depends on the initial misregistration $d_{0}$ and the same phase modulation $\Psi(x)$ can produce very different appearances based on $d_{0}$. An example of this effect is shown in Fig. 4. The halftone image in the example was assumed to have a constant area coverage of $50 \%(\mu=0.5)$. Figure $4(\mathrm{a})$ shows the phase modulation pattern $\Psi(x)$ and Fig. 4(b) shows the demodulated reflectance for this overlay for $d_{0}$ varying between 0 and 0.5 halftone cell units. For any given displacement $d_{0}$, the reflectance along the corresponding horizontal line through Fig. 4(b) defines the reflectance profile (along the $x$ direction) observed in the overlay constructed in the watermark detection process. It can be seen that the recovered patterns are scaled inverted versions of the modulation pattern for $d_{0} \in[0,0.25)$, scaled versions for $d_{0} \in(0.25,0.5]$, and for $d_{0}=0.25$, the recovered pattern is a constant gray-level region except in the boundary regions so that only the transitions would be seen.

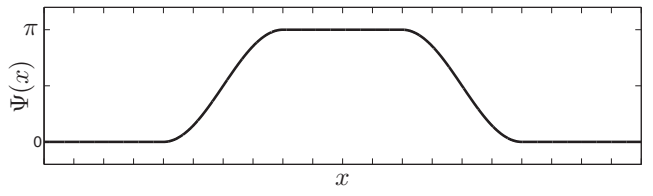

(a) Phase modulation signal

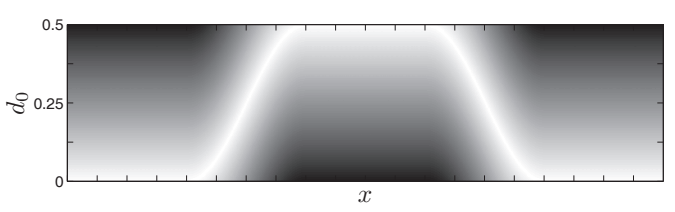

(b) Overlay reflectance

Fig. 4. Subfigure (b) shows the overlay reflectance corresponding to the modulating signal in (a) as a function of the misregistration displacement $d_{0}$.

\section{EXPERIMENTAL RESULTS}

An experimental evaluation of multiplexed clustered-dot halftone watermarking using the proposed bi-directional phase modulation and detection was conducted. Our setup utilized an electrophotographic printer with an addressability of $1200 \times 1200 \mathrm{dpi}$. The magnitudes of the frequencies $\left|\mathbf{f}_{1}\right|$ and $\left|\mathbf{f}_{2}\right|$ were chosen approximately 71 lines per inch (lpi) and orientation angles $\phi_{1}$ and $\phi_{2}$ were set to 45-deg. The contone Chapel image shown in Fig. 5(a) was used as the cover image and the watermark patterns with IEEE ICIP and Hong Kong logos as shown in Figs. 5(b) and (c) were embedded in the halftone image using bi-directional phase modulation. The printed image was generated in an 8 in $\times 8$ in format.

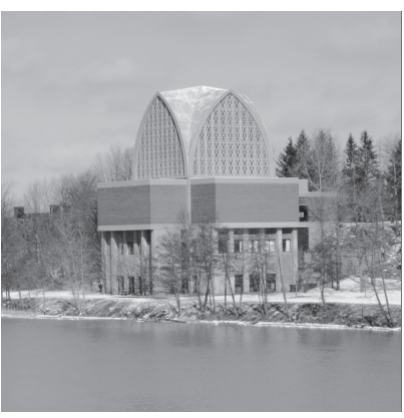

(a) Contone Chapel image

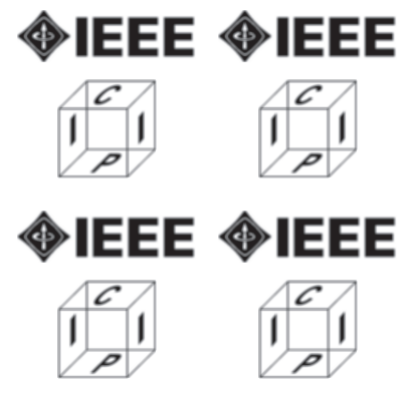

(b) $w_{1}(x, y)$

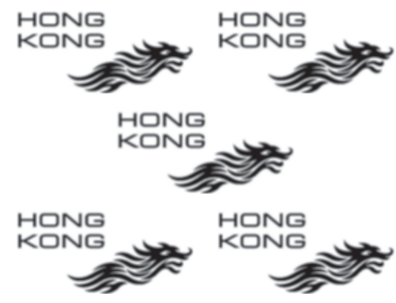

(c) $w_{2}(x, y)$
Fig. 5. Contone cover image and the embedded watermark patterns.

The printed halftone was scanned with a flatbed scanner with optical resolution of $1200 \times 1200$ dpi. The watermark patterns were revealed by superposing the scanned image with the corresponding 
line screen decoder masks. Figure 6 shows the detection results obtained for our example. The watermark patterns are clearly visible in the resulting overlays. However, as shown in Fig. 4, due to the local variations in the registration between the decoder mask and the watermarked halftone, the reflectance of the detected watermark patterns in Fig. 6 also varies: being either lighter or darker than the background or being visible only in the transition edge regions. Figure 7 demonstrates this via a digital simulation where misregistrations $d_{0}=0,0.25$, and 0.5 in halftone cell units are considered between the cover image halftone and the decoder mask. As shown in Fig. 4, the recovered watermark pattern corresponding to these misregistrations is either scaled inverted versions of the watermark patterns as shown in Fig. 7(a), a version in which only the boundaries corresponding to the transitions are seen as shown in Fig. 7(b), or scaled versions as shown in Fig. 7(c). The detected watermark patterns shown in Fig. 6 exhibit a blend of these three instantiations.

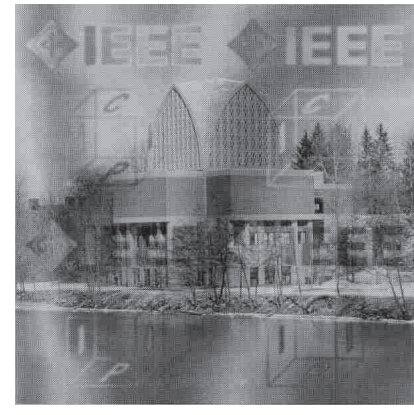

(a) $\hat{w}_{1}(x, y)$

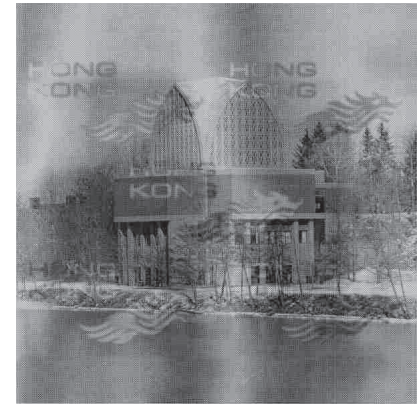

(b) $\hat{w}_{2}(x, y)$
Fig. 6. Detected watermark patterns. Artifacts due to re-screening in the printing process may not allow clear observation of the detected watermark patterns in the printed version of this document. Please refer the electronic version to clearly observe the detection results.
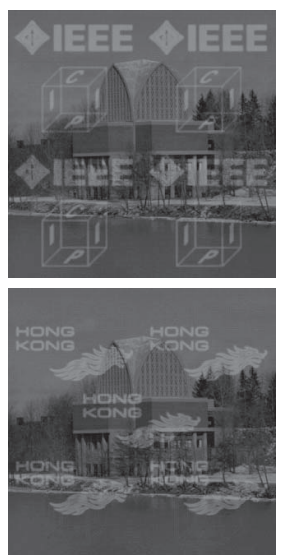

(a) $d_{0}=0$
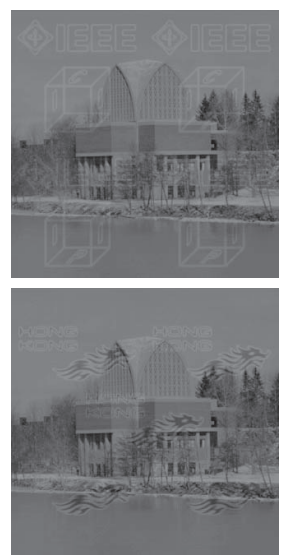

(b) $d_{0}=0.25$
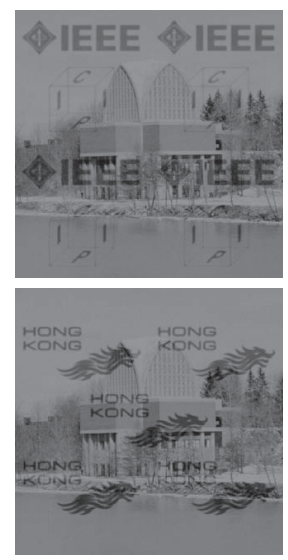

(c) $d_{0}=0.5$
Fig. 7. Dependence of the detected watermarks on thespatial misregistration between the watermarked image and the decoder masks. Subfigures (a), (b), and (c) show demodulated watermark patterns for misregistration displacements $d_{0}=0,0.25$, and 0.5 , respectively.

\section{CONCLUSION}

In this paper, we propose multiplexed clustered-dot halftone watermarks using bi-directional phase modulation and detection. Two in- dependent visual watermark patterns are embedded in the same region of a single hardcopy print with minimum visual impact. The watermark patterns are independently detected by overlaying the printed image, or scan thereof, with corresponding decoder masks. We also analyze the halftones with the embedding and demonstrate that the low frequency components that determine the visual appearance are unchanged by the modulation process . By combining this frequency domain analysis with spatial domain analysis, we also characterize the watermark detection process, which helps explain the observations in the detection. Experimental results demonstrate the efficacy of our methodology. The proposed method is general for clustered-dot halftones with arbitrary periodicities. In fact, using the method of [17], it can also be extended to color on a per colorant channel basis, allowing for embedding 8 independent watermark patterns in a rotated clustered-dot CMYK color halftone image.

\section{REFERENCES}

[1] C. M. Briquet, Les Filigranes: Dictionaire Histoire Des Marques Due Papier Des Leur Appartion Vers 1282, Jusquen 1600, 2nd ed. Leipzig, 1923.

[2] R. L. van Renesse, Optical Document Security, 3rd ed. Boston, MA: Artech House, 2005.

[3] R. Ulichney, Digital Halftoning. Cambridge, MA: MIT press, 1987.

[4] G. Sharma and S. Wang, "Show-through watermarking of duplex printed documents," in Proc. SPIE: Security, Steganography, and Watermarking of Multimedia Contents VI, E. J. Delp and P. W. Wong, Eds., vol. 5306, Jan. 2004, pp. 670-684.

[5] S. Huang and J. K. Wu, "Optical watermarking for printed document authentication," IEEE Trans. Info. Forensics and Security, vol. 2, no. 2, pp. 164-173, Jun. 2007.

[6] B. Oztan and G. Sharma, "Continuous phase-modulated halftones," IEEE Trans. Image Proc., vol. 18, no. 12, pp. 2718-2734, Dec. 2009.

[7] C. Liu, S. Wang, and B. Xu, "Authenticate your digital prints with Glossmark images," in Proceedings IS\&T NIP20: International Conference on Digital Printing Technologies, Oct. 2004, pp. 312-316.

[8] M. S. Fu and O. C. Au, "Correlation-based watermarking for halftone images," in Proc. IEEE Intl. Symp. on Circ. and Sys., May 2004, pp. 21-24.

[9] H. Z. Hel-Or, "Watermarking and copyright labeling of printed images," J. Electronic Imaging, vol. 10, no. 3, pp. 794-803, Jul. 2001.

[10] O. Bulan, V. Monga, G. Sharma, and B. Oztan, "Data embedding in hardcopy images via halftone-dot orientation modulation," in Proc. SPIE: Security, Forensics, Steganography, and Watermarking of Multimedia Contents X, E. J. Delp, P. W. Wong, J. Dittmann, and N. D. Memon, Eds., vol. 6819, Jan. 2008, pp. 68 190C-1-12.

[11] C. M. Hains, S. Wang, and K. T. Knox, "Digital color halftones," in Digital Color Imaging Handbook, G. Sharma, Ed. Boca Raton, FL: CRC Press, 2003, chapter 6.

[12] R. J. Pellar and L. Green, "Electronic halftone generator," United States Patent No. 4149183, 1979.

[13] R. J. Pellar, "Electronic halftone generator," United States Patent No. 4196451, 1980.

[14] B. Oztan, G. Sharma, and R. P. Loce, "Misregistration sensitivity in clustered-dot color halftones," J. Electronic Imaging, vol. 17, no. 2, pp. $023004,1-30$, Apr./Jun. 2008.

[15] D. Kermisch and P. Roetling, "Fourier spectrum of halftone images," J. Opt. Soc. Am., vol. 65, no. 6, pp. 716-723, Jun. 1975.

[16] I. Amidror and R. D. Hersch, "Fourier-based analysis of phase shifts in the superposition of periodic layers and their moiré effects," $J$. Opt. Soc. Am. A, vol. 12, no. 5, pp. 974-987, May 1996.

[17] B. Oztan and G. Sharma, "Per-separation clustered-dot color halftone watermarks: Exploiting differences in spatial periodicity and colorant spectra," J. Electronic Imaging, submitted for review Jan. 2010. 